\title{
Spondyloepimetaphyseal Dysplasia, Sponastrime Type
}

National Cancer Institute

\section{Source}

National Cancer Institute. Spondyloepimetaphyseal Dysplasia, Sponastrime Type. NCI

Thesaurus. Code C129031.

A rare disorder characterized by severe short stature, lumbar lordosis, midface

hypoplasia, micromelia, frontal bossing, epiphyseal and metaphyseal abnormalities. The inheritance is autosomal recessive. 